\title{
Recurrent method for constructing fuzzy cognitive maps for food security assessment
}

\author{
Aleksey Rogachev ${ }^{1,2, *}$ and Elena Melikhova ${ }^{1}$ \\ ${ }^{1}$ Volgograd State Agrarian University, Department of Mathematical Modeling and Informatics, \\ Volgograd, av. Universitetsky, 26, Russian Federation \\ ${ }^{2}$ All-Russian research Institute of irrigated agriculture, Volgograd, str. Timiryazeva, 9, Russian \\ Federation
}

\begin{abstract}
The advantages and limitations of algorithms and tools for supporting the procedure for assessing the level of regional food security based on a cognitive approach based on the construction of fuzzy cognitive maps (FCM) are considered. The results of the analysis of basic problems that are effectively solved using the mathematical apparatus of FCM research and problems that arise in this case are presented. The functional capabilities of tools that support the procedure for assessing the level of food security are analyzed. The article presents a method for recurrent construction of $\mathrm{NC}$ by consistently adding new concepts and re-evaluating the value of their mutual influence by experts. Recommendations on the choice of tools for solving the problem of assessing the level of regional food security, taking into account a number of economic and organizational groups of factors, are presented.
\end{abstract}

\section{Introduction}

Food security (FS) is a socio-economic category that can be evaluated by a complex integral indicator, the numerical assessment of which is not only ambiguous, but also controversial [1-4]. There are many approaches for its assessment, including expert, analytical, statistical, and other approaches, as well as corresponding hardware and software tools for their implementation, which to a certain extent allow us to numerically assess the value of this socio-economic category. There is no single generally accepted methodology for assessing the level of food safety. This is due to the complexity of the problem, the absence of a universal scale, the significant dimension of the problem of numerical estimation of FS, its insufficient structure, and not all the required numerical data available. To obtain an objective assessment of the current FS level and identify trends in its change, it is possible to use a cognitive approach based on the construction of fuzzy cognitive maps (FCM) [5-9].

Among the typical tasks successfully solved with the use of FCM, there is a possibility (V. Volkova, G. Gorelova, A. Kolodenkova, P. Ginis, V. Kamaev, A. Kulinich, P. Terelyansky, etc. $[3,9])$ to identify trends in the evolution of socio-economic systems for various purposes, including their controlled development. At the same time, there are

\footnotetext{
*Corresponding author: $\underline{\text { rafr@mail.ru }}$
} 
limitations to the use of cognitive modeling. In connection with the above, the adaptation and software implementation of the cognitive approach in relation to the numerical assessment of the FS level is an actual scientific and methodological problem.

\section{Materials and methods}

The research is based on such fundamental methods and approaches of system analysis as the system approach and systems theory, cognitive and graph-analytical analysis, the theory of algorithms, including the structure of recurrent algorithms, comparative and temporal analysis.

Note that recurrence is a method for calculating a function. A recurrent algorithm defines a method for calculating the terms of a sequence describing a function using recurrent formulas. The next member of the sequence is calculated as a function of the previous one:

$$
X k=f(X k-1)
$$

where $\mathrm{X} 0=\mathrm{a}$.

Note that a more complex case is possible when the next term of a recurrent number sequence depends not on one, but on two previous ones:

$$
X k=f(X k-1, X k-2) \text { where } X 0=a, X 1=b .
$$

In the theory of algorithms, when programming a recurrent algorithm, a recursive loop is organized that calculates the value of the next member of the sequence (1).

Despite the existence of a national food security Doctrine, FS provision, as a typical socio-economic system, is poorly structured [10]. For such systems, it is typical that their parameters and laws of behavior are described mainly at the qualitative level, and changes in their parameters can lead to difficult to predict changes in behavior. Modeling and managing such systems using traditional approaches based on analytical description or statistical observation is difficult. This forces us to resort to subjective approaches based on expert information processed using "common sense" logic, intuition, and heuristic approaches.

One of the most effective approaches is cognitive modeling based on special graph structures - cognitive maps. It allows the decision-maker to activate the intellectual processes and display the idea of the problem situation in the form of a formal model. Such a model is a cognitive map (CM), which is a set of factors on which a set of cause-andeffect relationships is set. A further development of the CM was the fuzzy cognitive maps (FCM), which include the weights of the concepts taken into account and characterize their mutual influence.

\section{Results}

Let's consider the possibilities of modifying the construction of the NCC based on the analysis of the problem aspects of cognitive modeling on the example of the FS level assessment. The main problems of the cognitive approach include the complexity of verifying both the NCC itself and modeling the evolution of SES using them.

There is a known approach to the verification of the NCC, which consists in identifying potentially possible systematic errors made during its construction. At the same time, the following threats (risks) are possible: 
- uncertainty in choosing the optimal number of concepts and significant connections between them;

- false definition of cause-and-effect relationships between concepts (false transitivity);

- incorrect logical and mathematical description of relationships between concepts;

- conditionality of dimensionless time when modeling the processes of system evolution.

Various software systems are known to support cognitive modeling processes-from the oldest foreign FCMappers [11] to domestic developments "IGLA", "Strela", "Strategist", etc. With similar capabilities, they differ mainly in the interfaces themselves, as well as in the technologies for organizing interaction with users.

An effective approach to FCM verification is to provide an explanation of how the result was obtained. In particular, the Russian "Canvas" system provides a special block for explaining the forecast, which generates a report on the step-by-step sequence of obtaining the result.

If necessary, after verification of the card, the FCM is corrected. There is a known method for correcting the NCC based on the analysis of the structural stability of the cognitive map. The essence of the method is to link the stability of the graph representing the map with its structure, but it does not provide unambiguous solutions for correcting the structure of the digraph. To increase the stability of the digraph, analytical work is supposed to correct its structure.

In the FCMappers system [11], you can set the initial weights of the concepts to be considered. The system allows you to visualize the NCC using the Pajek tool [12]. The Russian software system IGLA (an Intelligent Generator of the Best Alternatives) provides support for collective construction, research and adjustment of the FCM, calculation of its system indicators, as well as pulse dynamic modeling and scenario analysis.

The analytical review has shown that there are a number of different tools that provide cognitive modeling using NCC. Their choice is determined by the nature of the research task and the features of the simulated SES. therefore, researchers are often forced to create their own software prototypes $[6,13]$ that take into account the specifics of specific tasks.

Extending the recurrent approach to $\mathrm{n}$ considered members of the defined sequence, we can write:

$$
X k=f(X k-1, X k-2, \ldots, X k-n) \text { where } X 0=a, \ldots X n=z
$$

Let's take a closer look at the authors' proposed application of this approach to building fuzzy cognitive maps using the example of food security assessment.

Previously, it is necessary to build a ranked list of concepts that, according to experts, should be taken into account a priori when constructing a fuzzy cognitive map. By consistently adding the following concept from the a priori ranked list to the cognitive map, it is necessary to review the values of the weights corresponding to the new configuration of the modified graph of the formed cognitive map by expert means.

An outline algorithm of the proposed recursive approach to the construction of fuzzy cognitive maps is presented in Fig. 1.

Block 1 represents the initialization process unit 2 - the termination condition of the recursive procedures for adding concepts unit 3 - adding concepts in the IAC and expert estimation of all weights, block 4 - a simulation analysis of the NCC after adding a new concept. The recursive procedure is completed (block 5) when the condition of block 2 is met, after which a detailed scenario analysis can be performed.

\section{Discussion}


To solve the last of the key problems inherent in cognitive modeling listed above, according to Prof. G. Gorelova (Southern Federal University), regression dependencies can be applied [9]. The use of functional dependencies for setting the values of the influence of concepts on each other should be considered relevant. At the same time, a separate problem is not only the software implementation of this approach, but also the rationale for the rational choice of factors taken into account.

To implement this approach, you may need to create specialized hardware and software systems.

The " system for monitoring crisis situations in the social sphere "(RF patent for PM No. 59285) contains the following blocks: reception of expert requests 1 , reception of data on the level of social tension 2, reception of database records 3 servers, selection of addresses 4, identification of information sources 5, calculation of integrated socioeconomic tension 6, selection of interviewing cycles of information sources 7 of the mentioned tension (Fig. 2A).

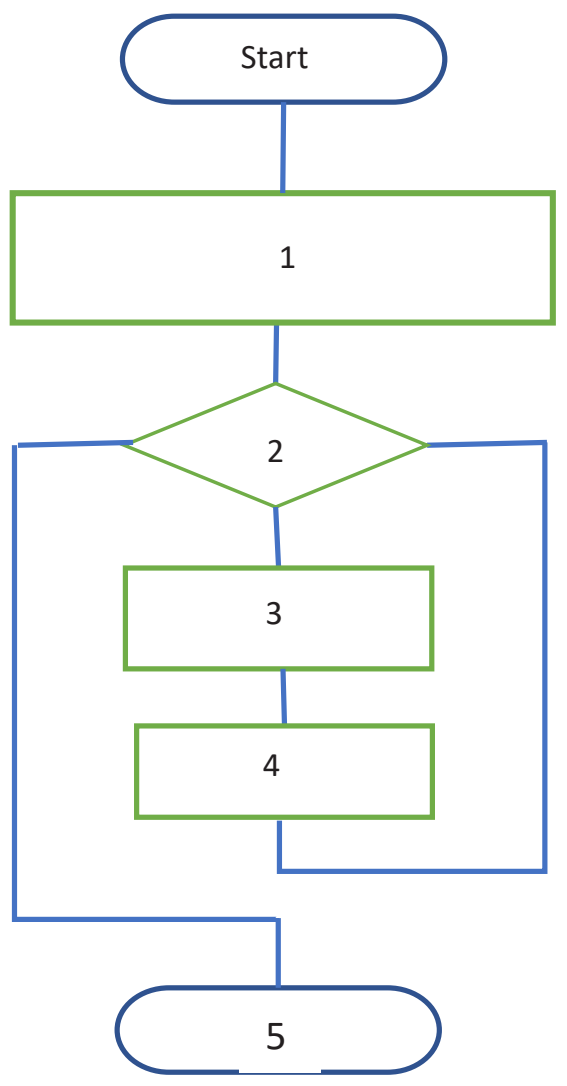

Fig. 1. High-level algorithm implementation recursive approach to the construction of fuzzy cognitive maps

A modified technical solution is also known (Pat. RF 2305319), which includes the following blocks): receiving plan records, receiving requests from users, receiving database server plan records, selecting the reference address of the plan and reading the address selection, forming the current address of the plan, adders (1st and $2 \mathrm{nd}$ ), forming the address of the time period of analysis, forming the addresses of database servers, managing writing and reading, and displaying block information (Fig. 2B). 
It is possible to predict the appearance of new technical solutions that develop the software and hardware complexes presented in Fig. 2.

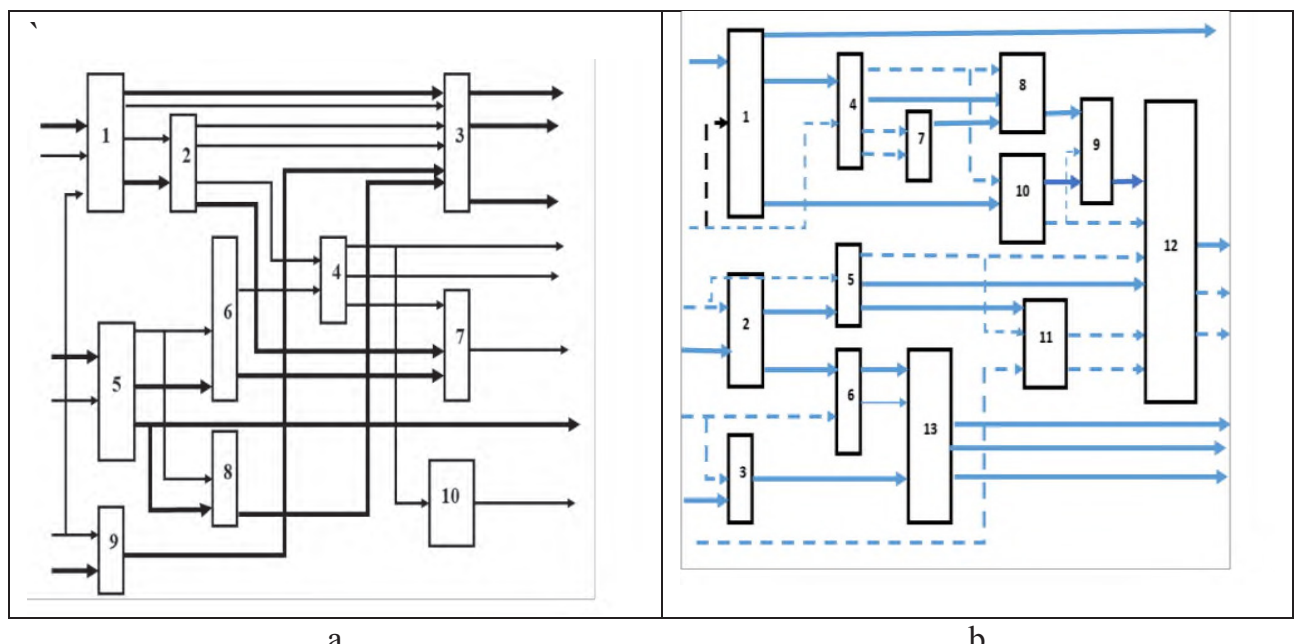

b

Fig. 2. Hardware and software for cognitive modelling: a - patent RU No. 59285; b - patent RU 2305319.

\subsection{Summary}

1. The constructed optimization model in the form of a linear programming problem (RLP) allows to justify the optimal distribution of limited resources for the simulated fields on irrigated areas of fields with different crops.

2. When solving the optimization problem, it is possible to determine the maximized values of the specific efficiency of agricultural production in energy units per unit area with different combinations of technical and energy coefficients, the volume of resources, the years of moisture supply and other factors.

This article was prepared with the financial support of the Russian Foundation for basic research for the project 19-07-01132.

\section{References}

1. E. Antamoshkina, A. Rogachev, Methodical approach to food security assessment and forecasting, Advances in economics, business and management research. Proceedings of the Volgograd State University International Scientific Conference "Competitive, Sustainable and Safe Development of the Regional Economy" (CSSDRE 2019) 184-187 (2019)

2. H.P. Weikard, Phosphorus recycling and food security in the long run: a conceptual modelling approach, Food Security 8, 2, 405-414 (2016)

3. G. Korshunov, V. Lipatnikov, A. Shevchenko, Decision support systems for information protection in the management of the information network. Fuzzy Technologies in the Industry - FTI 2018. Proceedings of the II International Scientific and Practical Conference 418-426 (2018) 
4. A. Rogachev, E. Antamoshkina, Mathematical modeling of the food-security level using a fuzzy cognitive approach IOP Conference Series: Earth and Environmental Science 403 (2019) Doi: 10.1088/1755-1315/403/1/012181

5. M. Skvortsova, V. Terekhov, Study of the possibility of applying intellectual methods in creating risk analysis decision support system in the socio-economic sphere, Proceedings of the 2019 IEEE Conference of Russian Young Researchers in Electrical and Electronic Engineering, ElConRus 1892-1896 (2019)

6. A. Rogachev, E. Antamoshkina, E. Melikhova, T. Pleschenko, Fuzzy cognitive modeling of the integral level of regional food security, Lecture Notes in Networks and Systems 129 LNNS 1173-1181 (2020)

7. K. Chrysafiadi, M. Virvou, A Knowledge Representation Approach Using Fuzzy Cognitive Maps for Better Navigation Support in an Adaptive Learning System, Springer Plus 2:81, 1-13 (2013)

8. R. Isaev, A. Podvesovskii, Generalized Model of Pulse Process for Dynamic Analysis of Sylov's Fuzzy Cognitive Maps CEUR Workshop Proceedings of the Mathematical Modeling Session, International Conference Information Technology and Nanotechnology (MM-ITNT 2017) 1904 7-63 (2017)

9. L. Ginis, G. Gorelova, A. Kolodenkova, Cognitive and simulation modeling of regional economic system development, International Journal of Economics and Financial Issues 6, S5, 97-103 (2016)

10. CR.J.K. Knight, D.J.B. Lloyd, A.S. Penn, Linear and Sigmoidal Fuzzy Cognitive Maps: An Analysis of Fixed Points URL: https://pdfs.semantiescholar.org/ (01.07.2018)

11. FCMappers. http://www.fcmappers.net/joomla/ (05.07.2018)

12. Pajek: analysis and visualization of large networks. URL: http://pajek.imfm.si/

13. A. Rogachev, E. Melikhova, E.N. Antamoshkina, Assessment and forecasting of agricultural production and food security based on fuzzy cognitive mathematical models, Proc. of the Lower Volga Agro-University Comp. 397-406 (2019) DOI: 10.32786/2071-9485-2019-04-49

14. O. Osoba, B. Kosko, Fuzzy Cognitive Maps of Public Support for Insurgency and Terrorism, Journal of Defense Modeling and Simulation: Applications, Methodology, Technology 14 (1), 17-32 (2017)

15. E. Gavriliuk, S. Mantserov, Fuzzy reliability model of systems for decision support in technical diagnostics. Fuzzy Technologies in the Industry - FTI 2018. Proceedings of the II International Scientific and Practical Conference 222-234 (2018)

16. M. Shcherbakov. V. Kamaev, A. Brebels et al. Outlier detection and classification in sensor data streams for proactive decision support systems, Journal of Physics: Conference Series 803, 1.012143 (2017) 